\title{
Genes for peripheral neuropathy and their relevance to clinical practice
}

\section{Donaghy}

\section{What does the future hold for genetic testing of peripheral neuropathies?}

1 dentification of neurological disease genes has expanded and transformed the neurologist's nosology in the last decade. Yet some clinicians see the resultant overload of detail as merely making the diagnosis and management of patients more cumbersome with little tangible benefit. Using the example of inherited peripheral neuropathy it is timely for a clinician's perspective of where neurogenetics has taken present day neurological practice and what the future might hold.

Prior to molecular genetics, most neurologists kept a simple classification of inherited peripheral neuropathy in mind: demyelinating and axonal forms of hereditary motor and sensory neuropathy (HMSN), also known as Charcot-Marie-Tooth disease (CMT) $i^{1}$ the hereditary sensory and autonomic neuropathies (HASN); ${ }^{2}$ hereditary liability to pressure palsies; ${ }^{3}$ and the hereditary amyloiditic polyneuropathies. ${ }^{4}$ Although workaday, this classification's deficiencies were apparent. For example, a significant minority of patients with HMSN could not be categorised cleanly as either Type 1 (demyelinating) or Type 2 (axonal) on the basis of electrophysiology, leading to the notion of intermediate forms. ${ }^{5}$ A range of severe HMSN-recessively inherited and affecting infants and children, and including the category known as congenital hypomyelinating neuropathyseemed to evade consistent classification. ${ }^{6}$ Our inability to make definitive diagnoses for these rare infantile disorders was particularly distressing given the family implications, profound motor disability, and sometimes death, which could result. So, when the chromosome 17 reduplication of the PMP-22 gene in CMT was described, a new dawn promised accurate diagnosis of genetic neuropathy and its implications.

Since that first flush of promise, everything has become complicated by detail. There are more genetic neuropathies than we had ever imagined, and philately seems as useful as neurology for practising this subspecialty. We should be grateful to colleagues who have taken the trouble to distil this genetic literature and provide us with contemporary classifications. ${ }^{7-10}$ A brief look at their contemporary classifications for HMSN-now named CMT again-shows 37 identified genes or loci. Of these, 22 reflect demyelinating forms, 13 axonal and 2 intermediate, with a range of autosomal dominant and recessive, and sex-linked recessive transmission. At last the causes of the severe childhood and congenital hypomyelinating forms are emerging. ${ }^{7-10}$ The deluge continues apace. New interesting forms of CMT associated with mutations of the lamin $\mathrm{A} / \mathrm{C}$ gene that encodes a nuclear envelope protein have been described, ${ }^{11}$ and of the gangliocideinduced differentiation-associated protein 1 (GDAPl). ${ }^{12}$ Also, different mutations affecting the same gene can produce demyelinating, intermediate or axonal forms of CMT-examples being CMTIB (myelin protein $\mathrm{P}_{0}$ mutations) and GDAPl mutations. Reviews get out of date quickly - the website http:// molgen-www.uia.ac.be/cmtmutations/ provides a real time encyclopaedia.

A similarly burgeoning range of genetic abnormalities seems to underlie other types of genetic neuropathy; acromutilating neuropathies of the HSAN type have been associated with the genetic locus for CMT Type 2B, or with the serine palmitoyltransferase long-chain base subunit l (SPTLC1) gene; the Riley-Day syndrome of familial dysautonomia is associated with mutations in the IkB kinase complexassociated protein (IKAP) gene; and selective neuropathies affecting small myelinated and unmyelinated fibres are associated with mutations in the tyrosine kinase A receptor for nerve growth factor (TRKA) gene. Memorising all of this is near impossible, even for academic clinicians subspecialising in peripheral neuropathy. The numerical classification has reached almost ridiculous proportions, whilst biochemical classifications have limited appeal for the non-cognoscenti.

Identification of these myriad genetic abnormalities underlying CMT has led to one surprising, but important intellectual realisation. At the beginning of this voyage of molecular genetic discovery, many had anticipated that each new gene would add a logical piece to the jigsaw of understanding the development of peripheral nerve and the maintenance of its structure. However, in reality no all-embracing view of the biology of peripheral nerve development and structure has emerged from this wide range of naturally occurring human mutations. Indeed, few mutations seem to affect processes or proteins unique to peripheral nerves. Most seem to affect functions likely to be fundamental to the biology of the cells of many different tissues. Even in the case of mutations affecting the protein responsible for peripheral nerve myelin compaction-the myelin protein zero (MPZ) gene-we find that the 80 different point mutations cause a bewildering array of CMT Type IB (demyelinating), CMT Type 2 (axonal), forms intermediate between CMTl and CMT2, congenital hypomyelinating and childhood onset forms of CMT, and a late onset form of progressive demyelinating neuropathy with features resembling chronic inflammatory demyelinating polyneuropathy. ${ }^{13}$ Clinically similar phenotypes of CMTl (demyelinating) can be associated with abnormalities of the various genes for the growth arrest protein peripheral myelin protein 22 (PMP-22), early growth response element 2 (EGR2), ganglioside-induceddifferentiation associated-protein-1 (GDAP1), myotubularin-related-protein-2 (MTMR2), n-myc-downstreamregulated-gene-1 (NDRG1), epithialgrowth-factor-related-protein-2 (EGR2), and periaxin (PRX). ${ }^{8}$

Thankfully the inability to develop or maintain an axon in CMT2 is founded in straightforward neurobiological logic when due to mutations in the genes for kinesin-motor-protein-1-B (KIFIB $\beta$ ) and neurofilament light chain (NFL), which involve axonal transport motor proteins and axonal structural intermediate filament proteins, respectively. But why do you get autosomal recessive CMT2 with one mutation of the laminin $\mathrm{A} / \mathrm{C}$ nuclear envelope protein whilst other mutations produce the EmeryDreyfus muscular dystrophies, limb girdle muscular dystrophies, cardiomyopathy, and partial lipodystrophy ${ }^{8}$ Molecular genetic analysis of diseases has taught us something of the specific biology maintaining the structure of the peripheral nervous system, but it

\section{Abbreviations: CMT, Charcot-Marie-Tooth disease; HASN, hereditary sensory and autonomic neuropathy; HMSN, hereditary} motor and sensory neuropathy 
has told us rather more about the vulnerability of neurones to seemingly more fundamental aberrations of cell biology.

Present day clinical practice has not been revolutionised by molecular genetic tests for peripheral neuropathy. Of course, such tests can provide patients with diagnosis couched in fact rather than diagnostic opinion; not unimportant in an era when scientific explanations dominate medical thinking. More confident prediction of prognosis and risk to offspring can follow accurate genetic diagnosis, but these were reasonably accurate previously given the defined clinical nature of most neuropathies and their clear inheritance patterns. In an era of ever smaller sibships, molecular genetics can allow proof of diagnosis when there is no opportunity to look for another affected family member. One's experience in a peripheral neuropathy clinic shows that diagnostic genetic testing for HMSN does have its drawbacks. Routine molecular genetic testing is available for the $70 \%$ of HMSN due to reduplication of the PMP-22 gene, and another $20 \%$ due to mutations of myelin protein $\mathrm{P}_{0}$ and Connexin 32 genes. However, standard HMSN1A, due to a PMP-22 reduplication, is pretty easily diagnosable on grounds of clinical, electrophysiological, and pedigree features. A positive genetic test often merely adds icing to the cake. To a lesser extent the same can be said of the sex-linked neuropathy due to Connexin 32 mutations, and some of the more common myelin protein $\mathrm{P}_{0}$ mutations. Molecular genetic testing is not routinely available for the panoply of rarer genetic forms of HMSN, at least not in the United Kingdom. What this leaves is a sense of frustration for the clinician because, although routinely available molecular genetic testing is good for proving a diagnosis of which you are already fairly sure on clinical grounds, it offers nothing in that $10 \%$ of patients where you are sure that they have HMSN, but the features are atypical for the main syndromes. Screening for PMP-22 and $\mathrm{P}_{0}$ in infants and children can help enormously in differentiating Dejerine-Sottas disease and congenital hypomyelinating neuropathy from potentially treatable chronic inflammatory demyelinating polyneuropathy.

What does the future hold for genetic testing of peripheral neuropathies?
Firstly, the difficulty of genetic diagnosis for the rarer and clinically more difficult syndromes would be revolutionised by the design of DNA chip, allowing simultaneous screening of a patient's DNA for all known CMT gene mutations. ${ }^{14}$ Second, considerable ethical questions will face the use of molecular genetics in prenatal diagnosis of patients and families at risk of neuropathy. For a few with the potentially lethal or severely disabling infantile and childhood forms, such diagnosis might allow parents to make a decision about selective abortion. However, for the vast majority of patients with genetic peripheral neuropathy, the disorder is only slowly progressive or relatively stable throughout life, without causing overwhelming disability, and generally without significantly affecting life expectancy. When the subject is discussed in a hypothetical manner with some of my HMSNIA patients, none of them have felt that their foot deformity and reduced athletic abilities have made them wish they had never been born. Third, the holy grail of molecular genetic testing, as outlined by its pioneers ${ }^{15}$ was to allow new genes to be flown into the body's cells to reverse the abnormality as early as possible to prevent permanent neural damage. The attraction of this remains as strong as ever, though the scientific methodology and commercial realisation remain far off. In the meantime, we should take heart from the reports of improvements in the CMT phenotype of animal models where overexpression of PMP-22 is modulated by administering ascorbic acid ${ }^{16}$ or progesterone receptor antagonists. ${ }^{17}$ One suspects that lower priority will apply to genetic modification of peripheral neuropathy than to some of the lethal or severely disabling genetic disorders of childhood. Nonetheless, the opening of such opportunities for all seems to have been signalled by the somewhat Orwellian UK White Paper, ${ }^{18}$ which has asked the Human Genetics Commission to report by the end of 2004 on "the case for screening babies at birth and storing information about their genetic profile for future use in tailoring healthcare according to their needs and their genetic make up."

J Neurol Neurosurg Psychiatry

2004;75:1371-1372.

doi: 10.1136/jnnp.2004.041939

Correspondence to: $\operatorname{Dr} M$ Donaghy, University Dept of Clinical Neurology, The Radcliffe
Infirmary, Woodstock Road, Oxford OX2 6HE, UK; joanna.wilkinson@clneuro.ox.ac.uk

\section{REFERENCES}

1 Harding AE, Thomas PK. The clinical features of hereditary motor and sensory neuropathy types I and II. Brain 1980;103:259-280.

2 Donaghy M, Hakin RN, Bamford JM, et al. Hereditary sensory neuropathy with neurotrophic keratitis. Description of an autosomal recessive disorder with a selective reduction of small myelinated nerve fibres and a discussion of the classification of the hereditary sensory neuropathies. Brain 1987; 110:563-583.

3 Earl CJ, Fullerton PM, Wakefield GS, et al. Hereditary Neuropathy, with Liability to Pressure Palsies; a Clinical and Electrophysiological Study of Four Families. Q J Med 1964;33:481-498.

4 Glenner GG, Murphy MA. Amyloidosis of the nervous system. J Neurol Sci 1989;94:1-28.

5 Davis CJ, Bradley WG, Madrid R. The peroneal muscular atrophy syndrome: clinical, genetic, electrophysiological and nerve biopsy studies. I. Clinical, genetic and electrophysiological findings and classification. J Genet Hum 1978;26:311-349

6 Gabreels-Festen AA, Joosten EM, Gabreels FJ, et al. Hereditary motor and sensory neuropathy of neuronal type with onset in early childhood. Brain $1991 ; 114: 1855-1870$

7 Berciano J, Combarros O. Hereditary neuropathies. Curr Opin Neurol 2003;16:613-622

8 Kuhlenbaumer G, Young P, Hunermund G, et al. Clinical features and molecular genetics of hereditary peripheral neuropathies. I Neurol 2000;249:1629-1650.

9 Pareyson D. Diagnosis of hereditary neuropathies in adult patients. J Neurol 2003;250: 148-160.

10 Reilly MM, Hanna MG. Genetic neuromuscular disease. J Neurol Neurosurg Psychiatry 2002;73:II12-21

11 Chaouch M, Allal Y, De Sandre-Giovannoli A, et al. The phenotypic manifestations of autosomal recessive axonal Charcot-Marie-Tooth due to a mutation in Lamin A/C gene. Neuromuscul Disord 2003;13:60-67.

12 Birouk N, Azzedine H, Dubourg O, et al Phenotypical features of a Moroccan family with autosomal recessive Charcot-Marie-Tooth disease associated with the S194X mutation in the GDAP1 gene. Arch Neurol 2003;60:598-604

13 Donaghy M, Sisodiya SM, Kennett R, et al. Steroid responsive polyneuropathy in a family with a novel myelin protein zero mutation. J Neurol Neurosurg Psychiatry 2000;69:799-805.

14 Pleasure DE. Genetics of Charcot-Marie-Tooth disease. Arch Neurol 2003;60:481-482.

15 Weatherall DJ. The new genetics and clinical practice. London: Nuffield Provincial Hospitals Trust, 1982

16 Passage E, Norreel JC, Noack-Fraissignes P, ef al. Ascorbic acid treatment corrects the phenotype of a model model of Charcot-MarieTooth disease. Nature Medicine 2004; 10:396-401.

17 Sereda MW, Meyer zu Horst G, Suter U, et al. Therapeutic administration of progesterone antagonist in a model of Charcot-Marie-Tooth disease (CMT-1A). Nature Medicine 2003;9:1533-1537.

18 Department of Health. Our inheritance, our future. Realising the potential of genetics in the NHS. Genetics White Paper, 2003. 
Embolic stroke

\section{Embolic stroke: the heart-brain connection reassessed}

\section{Brainin, C Stöllberger}

\section{Transesophageal echocardiography possibly underutilised in stroke?}

A utopsy series published a century ago, showed that cerebral emboli arising from the major vessels and the heart are an important pathophysiological cause of stroke. ${ }^{1}$ Since then not much has changed. According to one recent and comprehensive textbook ${ }^{2}$ embolism accounts for up to $60 \%$ of all ischemic strokes. Cardiac conditions associated with such cerebral emboli are atrial fibrillation in $45 \%$ of the cases, followed by acute myocardial infarction, ventricular aneurysm, rheumatic heart disease, prosthetic cardiac valves, aortic atheroma, and other causes.

With the availability of transesophageal echocardiography (TEE) in many stroke centres, intracardiac emboli resting in the left atrial appendage or left atrium can now be readily identified and treatment with anticoagulation can be installed. ${ }^{3}$ In this issue Sen et al ${ }^{4}$ (see page 1421) have reassessed the cardiac risk factors in a systematic study using TEE within a week of stroke onset in a largely unselected cohort of stroke patients. Their aim was to set up criteria for refinement of selection for TEE in stroke patients due to clinical or laboratory characteristics. Analysis of their consecutive 151 stroke patients showed that demographic factors, clinically assessable risk factors, as well as blood coagulation parameters did not differ between patients with and without a cardiac thrombus, whereas several other investigational findings were in favour of finding a cardiac thrombus by means of TEE-stroke appearance on CT/MRI compatible with a large vessel stroke, cardiac ischemia visible on ECG, and systolic left ventricular dysfunction.

What the authors found was only partly what they were looking for. Firstly, they found a $26 \%$ prevalence rate (40 out of 151 patients) of intracardiac thrombus (mostly in the left atrial appendage), which is higher than in most previous reports. The authors attribute this high rate to the routinely used multiplanar TEE technique (whereas the lower prevalence reported in previous studies had mostly used monoplanar or biplanar probes).

Secondly, Sen et al found a similarly high prevalence in lacunar type infarcts (24\%, 16 out of 66 patients), which implies that the presence of small artery infarcts on neuroimaging does not exclude the necessity to perform TEE in a considerable proportion of lacunar stroke patients.

One other frequently encountered clinical uncertainty comprises the necessity to perform TEE in stroke patients with atrial fibrillation. It is commonly held that the presence of atrial fibrillation obviates TEE due to the fact that anticoagulation would have been the therapy of choice anyway. Thus, it is argued that it is not necessary to add additional costs, discomfort, or a small but notable risk using a semi-invasive technique such as TEE. Furthermore, clinical findings of increased age and hypertension have been shown to be more useful than TEE findings to assess embolic risk in atrial fibrillation patients. ${ }^{5}$ Others might argue that it is simply important to localize, specify, and treat the cause of the stroke. The demonstration of an underlying pathology such as an intracardiac thrombus, the documentation of its size and location, plus any additional finding of cardiac structural abnormality or associated intra-cardiac pathology, might give additional weight to initiate anticoagulant therapy that otherwise might not have been indicated in such a mandatory way. The latter argument is supported by other reports, among them one by the same group ${ }^{6}$ that accumulates evidence to show the beneficial effect of anticoagulation on thrombus size reduction. Up until now, however, it has not been proven by any prospective study that stroke patients with intracardiac thrombi diagnosed by TEE have a higher recurrence rate than stroke patients without thrombi.

In spite of increasing awareness of the benefits of TEE in treating stroke patients there is still a need to do what this study has set out to achieve: to develop a clinically reliable set of criteria for investigating patients by means of TEE and to compare the results by looking for markers for urgency, intensity, or duration of anticoagulation. To do this properly a predefined and prospective series will have to be performed to validate these criteria and give some predictive rates. Until then the impression prevails that TEE is underused in acute stroke.

J Neurol Neurosurg Psychiatry 2004;75:1373. doi: 10.1136/jnnp.2004.046367

\section{Authors' affiliations}

M Brainin, Department Neurology

Donauklinikum and Centre Neurosciences,

Donau-Universität, Maria Gugging, Austria

C Stöllberger, 2nd Medical Department,

Krankenanstalt Rudolfstiffung, Vienna, Austria

Correspondence to: Professor Michael Brainin, Donauklinikum and Donau-Universität, Hauptstrasse 2, A-3400 Maria Gugging, Austria; michael.brainin@donau-uni.ac.at

\section{REFERENCES}

1 Chiari H. Über das Verhalten des Teilungswinkels der Carotis communis bei der Endarteriitis chronica deformans. Verh Dtsch Path Ges 1905;(9):326-332.

2 Furie KL, Homma S, Kistler JP. Cardiac Diseases. In: Mohr JP, et al, eds. 4th ed. Stroke. New York: Churchill Livingston, 2004:747-759.

3 The European Stroke Initiative Executive Committee and EUSI Writing Committee. European Stroke Initiative Recommendations for Stroke Management-Update 2003. Cerebrovasc Dis 2003;16:311-337.

4 Sen S, Laowatana S, Lima J, et al. Risk factors for intracardiac thrombus in patients with recent ischemic cerebrovascular events. J Neurol Neurosurg Psychiatry 2004;75:1421-5.

5 Stöllberger C, Chnupa P, Kronik G, Brainin M, Finsterer J, Schneider B, Slany J, for the ELAT Study Group. Transesophageal echocardiography to assess embolic risk in patients with atrial fibrillation. Ann Intern Med 1998; 128:630-638.

6 Sen S, Lima JA, Oppenheimer SM. Changes in cardiac thrombus status after cerebral ischemia. Cerebrovasc Dis 2004;17:175-181. 


\section{Intravenous immunoglobulins: a treatment for Alzheimer's disease?}

\section{E Hack, P Scheltens}

\section{A novel therapeutic option for Alzheimer's disease}

A $\beta$-peptide is generally considered to play a central role in the pathogenesis of Alzheimer's disease $(\mathrm{AD})$. The peptide is cleaved from amyloid precursor protein (APP) by secretases and is a key component of the amyloid plaques. Amyloid plaques may also contain other proteins such as serum amyloid $\mathrm{P}$ component (SAP), activated complement proteins, clusterin, and $\alpha$ l-antichymotrypsin. Observations in mice carrying the human APP transgene support the importance of $A \beta$-peptide as a driving force for intracerebral amyloid deposits in $\mathrm{AD}$. The mechanisms leading to neurotoxicity and neurodegeneration induced by $\mathrm{A} \beta$-peptide are not yet clear. According to one scenario deposits of $\mathrm{A} \beta$-fibrils, together with associated proteins, are toxic for neurones-either directly or indirectly-by recruitment and stimulation of microglial cells. An alternative scenario claims a major role of $A \beta$-oligomers as mediators of neurotoxicity. ${ }^{1}$ Clearance of intracerebral amyloid deposits is currently one of the therapeutic options under investigation for $\mathrm{AD}$.

Recently it was found that vaccination with $A \beta$-peptide slowed down the amyloid accumulation in the brains of APP transgenic mice, ${ }^{2}$ the effect of which could be reproduced by administration of anti-A $\beta$ antibodies. ${ }^{3}$ This has led to clinical studies on the effect of active immunisation of $\mathrm{AD}$ patients with $\mathrm{A} \beta$-peptide. Although promising results in some patients were observed, this active immunisation approach was accompanied by severe side effects-in particular, severe meningoencephalitis.

Specific antibodies against A $\beta$-peptide suitable for treatment in $\mathrm{AD}$ patients are not yet available. In this issue (pp 1472), Dodel and coworkers describe the effects of passive immunisation with intravenous immunoglobulins (IG) in five patients with $\mathrm{AD} .{ }^{4}$ Intravenous IG are prepared from plasma pools of thousands of normal donors, and initially were developed as a substitution therapy for immunodeficient patients. Later, intravenous IG was found to be beneficial in a number of inflammatory or immune disorders including some neurological diseases such as chronic inflammatory demyelinating polyneuropathy and possibly multiple sclerosis. ${ }^{5}$ Because healthy individuals have circulating auto-antibodies against A $\beta$-peptide, intravenous IG contains antibodies against $A \beta$-peptide as well. For this reason Dodel et al evaluated the effect of this drug in patients with AD. Total levels of $A \beta$-peptide decreased in cerebrospinal fluid (CSF) in the five patients evaluated, whereas levels of the peptide in serum increased. No effect on A $\beta 1-42$ levels were observed. In addition, stabilisation or even a mild improvement in cognitive function was observed in the patients.

These results raise a number of questions. Firstly, the number of patients studied is small and, therefore, as the authors also indicate, larger studies are needed to confirm that this treatment can stabilise or even improve cognitive functions in AD. Secondly, a dose of $0.4 \mathrm{~g}$ intravenous IG per $\mathrm{kg}$ body weight for three consecutive days every 4 weeks for 6 months was given-a regular dose used in immune disorders. Because the patients were given only one dose it is unclear whether this dose is optimal. Thirdly, the mechanism of action of intravenous $\mathrm{IG}$ in $\mathrm{AD}$ patients is not clear. The authors postulate that the effects are mediated by antibodies against $A \beta$-peptide, which indeed are present in intravenous IG. However, if these antibodies would mediate the effect, it is still not clear how they do so. Enhanced clearance of total $A \beta$ peptide is not supported by the increased levels in serum, which actually are more consistent with decreased clearance-at least in the periphery. On the other hand, the lower CSF levels support enhanced clearance in the cerebral compartment. However, it is puzzling why levels of $\mathrm{A} \beta \mathrm{l}-42$ did not change upon intravenous IG treatment because this peptide is considered to be the most pathogenic $\mathrm{A} \beta$-peptide in $\mathrm{AD}$. Therefore, if the beneficial effects of intravenous IG were due to the presence of anti-A $\beta$ antibodies, it would be more likely that they may have been related to neurones. A series of studies in vitro and in vivo on the effects of intravenous IG have shown that it has many other effects on immune and inflammatory reactions that may be relevant for its mode of action in AD. For example, intravenous IG has been shown to interfere with complement deposition onto targets. Indeed, amyloid plaques of $\mathrm{AD}$ contain activated complement proteins. Efficient inhibition of complement fixation to targets by intravenous IG occurs at higher doses of intravenous IG $(2 \mathrm{~g} / \mathrm{kg} / \mathrm{day})$ than those administered in the study by Dodel et al. Hence, if the complement modulating effect is the mechanism of action in $\mathrm{AD}$, it can be predicted that somewhat higher doses may even lead to better clinical effects. Another intriguing possibility is that intravenous IG may have affected the function of activated microglial cells surrounding the plaques. Animal experiments in rats have clearly shown that intravenous IG can stimulate macrophages and neutrophils via Fc receptors. ${ }^{6}$ Therefore, it is comprehensible that the brain microglial cells become stimulated upon intravenous IG administration, leading to enhanced clearance of amyloid deposits. Furthermore, changes in cytokine levels have been repeatedly found in response to intravenous IG administration. Hence, one could also postulate that the effects of intravenous $\mathrm{IG}$ in the $\mathrm{AD}$ patients described by Dodel et al were due to altered cytokine production by microglial cells.

Definite conclusions regarding the use of intravenous IG in $\mathrm{AD}$-with respect to both the clinical effects as well as to the mode of action - cannot be made on the basis of the study by Dodel and coworkers. However, the paper highlights a novel and interesting therapeutic option for $\mathrm{AD}$, which seems worthy to be explored in further studies. In addition, the effects of intravenous IG may need to be studied in the transgenic mouse models for $\mathrm{AD}$ to better understand the mechanism of action.

J Neurol Neurosurg Psychiatry

2004; 75:1374-1375.

doi: 10.1136/jnnp.2004.043141

\section{Authors' affiliations}

C E Hack, Department of Immunopathology, Sanquin Research at CLB, Amsterdam P Scheltens, Department of Neurology and Alzheimer Center, VU University Medical Center, 1007 HVAmsterdam, The Netherlands

Correspondence to: Professor P Scheltens, Department of Neurology and Alzheimer Center, VU University Medical Center, PO Box 7057, Amsterdam, 1007 HV, the Netherlands; p.scheltens@vumc.nl 


\section{REFERENCES}

1 Lambert MP, Barlow AK, Chromy BA, et al. Diffusible, nonfibrillar ligands derived from Abeta 1-42 are potent central nervous system neurotoxins. Proc Natl Acad Sci U S A 1998:95:6448-53.

2 Schenk D, Barbour R, Dunn W, et al. Immunization with amyloid-beta attenuates Alzheimer-disease-like pathology in the PDAPP mouse. Nature 1999;400: 173-7.

3 Bard F, Cannon C, Barbour R, et al. Peripherally administered antibodies against amyloid betapeptide enter the central nervous system and reduce pathology in a mouse model of Alzheimer disease. Nat Med 2000:6:916-9.

4 Dodel R, Du Y, Depboylu C, et al. Intravenous immunoglobulins containing antibodies against beta-amyloid for the treatment of Alzheimer's disease. J Neurol Neurosurg Psychiatry 2004;75: 1472-4.

5 Dalakas MC. Intravenous immune globulin therapy for neurologic diseases. Ann Intern Med 1997; 126:721-30.

6 Bleeker WK, Teeling JL, Verhoeven AJ, et al. Vasoactive side effects of intravenous immunoglobulin preparations in a rat model and their treatment with recombinant platelet-activating treatment with recombinant platelet-activating
factor acetylhydrolase. Blood 2000;95: 1856-61.

\section{Are multiple acute small subcortical infarctions caused by embolic mechanisms?}

\section{B Norrving}

\section{Multiple lacunar infarct mechanisms}

et this issue the paper by Chowdhury t al (see page 1416) ${ }^{1}$ is an important addition to the list of recent publications that challenge traditional concepts on the mechanisms of acute ischaemic stroke. According to conventional teaching, an acute ischaemic stroke is characterised by infarction confined to a single localised (focal) region of the brain. However, new neuroimaging techniques - in particular diffusion weighted magnetic resonance imaging (dw-MRI) - have modified this view.

In a recent study, ${ }^{2}$ scattered lesions in one vascular territory or multiple lesions in multiple vascular territories were actually more common than single lesions. Although dw-MRI is very sensitive in the ultra-early detection of cerebral ischaemia, it should be recognised that the dw-MRI lesions are not equivalent with infarction. Dw-MRI abnormalities may be reversible if the level of ischaemia is mild, or if early reperfusion occurs-for example, by means of thrombolytic therapy. Nevertheless, dw-MRI findings may give clues to underlying pathophysiology.

Scattered or multiple acute ischaemic lesion patterns have been associated with embolism from cardiac or large artery sources, ${ }^{2}$ which, in particular, should also be logically plausible if cortical territories are involved. However, in the present series of 10 patients with multiple infarcts mainly confined to subcortical regions, ${ }^{1}$ an embolic source was found in only one case. Could an embolic source have been over-looked? The possibility cannot be excluded because the patients were not investigated with trans-oesophageal ultrasound (which might have disclosed atherosclerotic aortic arch disease, for example); however, it appears unlikely.

The dw-MRI findings suggested that the subcortical lesions had occurred within several weeks rather than at exactly the same time. Also in this field, concepts have changed: the acute phase of ischaemic stroke is much more dynamic than previously thought. A recent dw-MRI study ${ }^{3}$ disclosed early recurrent lesions on neuroimaging in one third of all patients within the first week, while clinical recurrence was evident in only $2 \%$. Accepting that embolism is the unlikely cause in the present series, what other mechanisms may cause a clustering of multifocal cerebral ischaemia? At present, the answer is unknown but systemic factors like blood pressure regulation, haemorheological factors, infection, endothelial dysfunction, and possibly even stress might contribute. The present study underscores current gaps in the knowledge of precipitating causes of acute cerebral ischaemia-an under-investigated topic.

The present study also highlights recent insights that "silent" or "covert" cerebral infarcts are severalfold more common than ischaemic stroke, i.e. infarcts that present with the acute onset of focal neurological deficits. According to a recent analysis, less than $7 \%$ of all cerebrovascular lesions are associated with overt clinical symptoms, i.e. fulfilling the definition of stroke. ${ }^{4}$ Covent cerebrovascular lesions are important determinants for cognitive dysfunction, dementia, recurrent stroke, and death.

Recent technological advances have provided us with the tools for research into these issues. Progress in diagnosis and prevention of overt and covert cerebrovascular disease will require an open mind, ready to challenge traditional concepts of cerebrovascular disease.

J Neurol Neurosurg Psychiatry 2004;75:1375. doi: 10.1136/jnnp.2004.046748

Correspondence to: Dr B Norrving, Department of Neurology, Lund University Hospital, Lund S-22185, Sweden; bo.norrving@neurol.lu.se

\section{REFERENCES}

1 Chowdhury D, Wardlaw JM, Dennis MS. Are multiple acute small subcortical infarctions caused by embolic mechanims. J Neurol Neurosurg Psychiatry 2004;75:1416-20.

2 Kang DW, Chalela JA, Ezzeddine MA, et al. Association of ischaemic lesion patterns on early diffusion-weighted imaging with TOAST stroke subtypes. Arch Neurol 2003;60:1730-4.

3 Kang DW, Latour LL, Chalela JA, et al. Early ischemic lesion recurrence within a week after acute ischemic stroke. Ann Neurol 2003;54:66-74.

4 Leary MC, Saver JL. Annual incidence of first silent stroke in the United States: a preliminary estimate. Cerebrovasc Dis 2003; 16:280-85. 